\title{
The major upgrade of the MAGIC telescopes, Part I: The hardware improvements and the commissioning of the system
}

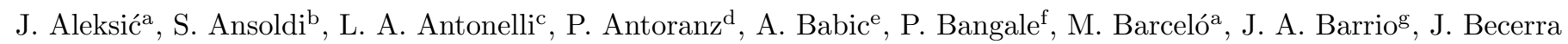
González $^{\text {h,aa }}$, W. Bednarek ${ }^{\mathrm{i}}$, E. Bernardini ${ }^{\mathrm{j}}$, B. Biasuzzi ${ }^{\mathrm{b}}$, A. Biland ${ }^{\mathrm{k}}$, M. Bitossi ${ }^{\mathrm{y}}$, O. Blanch ${ }^{\mathrm{a}}$, S. Bonnefoy ${ }^{\mathrm{g}}$,

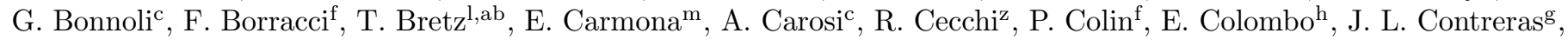

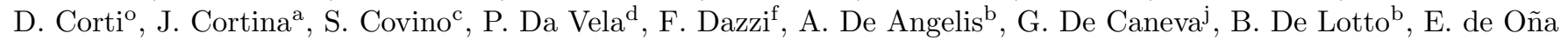

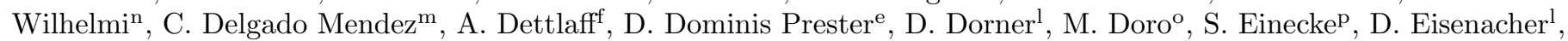
D. Elsaesser ${ }^{1}$, D. Fidalgo ${ }^{\mathrm{g}}$, D. Fink ${ }^{\mathrm{f}}$, M. V. Fonseca ${ }^{\mathrm{g}}$, L. Font ${ }^{\mathrm{q}}$, K. Frantzen ${ }^{\mathrm{p}}$, C. Fruck ${ }^{\mathrm{f}}$, D. Galindo ${ }^{\mathrm{r}}$, R. J. García López $^{\mathrm{h}}$, M. Garczarczyk ${ }^{\mathrm{j}}$, D. Garrido Terrats ${ }^{\mathrm{q}}$, M. Gaug ${ }^{\mathrm{q}}$, G. Giavitto ${ }^{\mathrm{a}, \mathrm{j}}$, N. Godinoviće ${ }^{\mathrm{e}}$, A. González Muñoz ${ }^{\mathrm{a}}$, S. R. Gozzini ${ }^{j}$, W. Haberer ${ }^{\mathrm{f}}$, D. Hadasch ${ }^{\mathrm{n}, \mathrm{ac}}$, Y. Hanabata ${ }^{\mathrm{s}}$, M. Hayashida ${ }^{\mathrm{s}}$, J. Herrera ${ }^{\mathrm{h}}$, D. Hildebrand ${ }^{\mathrm{k}}$, J. Hose $^{\mathrm{f}}$,

D. Hrupec e , W. Idec ${ }^{\mathrm{i}}$, J. M. Illa ${ }^{\mathrm{a}}$, V. Kadenius ${ }^{\mathrm{t}}$, H. Kellermann ${ }^{\mathrm{f}}$, M. L. Knoetigk , K. Kodani ${ }^{\mathrm{s}}$, Y. Konno ${ }^{\mathrm{s}}$, J. Krause ${ }^{\mathrm{f}}$,

H. Kubo ${ }^{\mathrm{s}}$, J. Kushida ${ }^{\mathrm{s}}$, A. La Barbera ${ }^{\mathrm{c}}$, D. Lelas ${ }^{\mathrm{e}}$, J. L. Lemus ${ }^{\mathrm{g}}$, N. Lewandowska ${ }^{1}$, E. Lindfors ${ }^{\mathrm{t}}$,ad, S. Lombardi ${ }^{\mathrm{c}}$,

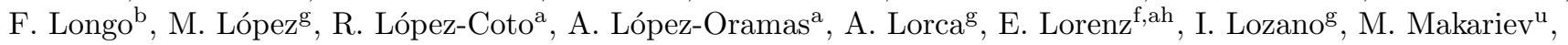
K. Mallot ${ }^{\mathrm{j}}$, G. Maneva ${ }^{\mathrm{u}}$, N. Mankuzhiyil ${ }^{\mathrm{b}}$ ae, K. Mannheim ${ }^{\mathrm{l}}$, L. Maraschic ${ }^{\mathrm{c}}$, B. Marcoter ${ }^{\mathrm{r}}$, M. Mariotti ${ }^{\mathrm{o}}$, M. Martínez ${ }^{\mathrm{a}}$, D. Mazin ${ }^{\mathrm{f}, *}, \mathrm{U}_{\text {. Menzel }}^{\mathrm{f}}$, J. M. Miranda ${ }^{\mathrm{d}}$, R. Mirzoyan ${ }^{\mathrm{f}}$, A. Moralejo $^{\mathrm{a}}$, P. Munar-Adrover ${ }^{\mathrm{r}}$, D. Nakajima ${ }^{\mathrm{s}}$, M. Negrello ${ }^{\circ}$,

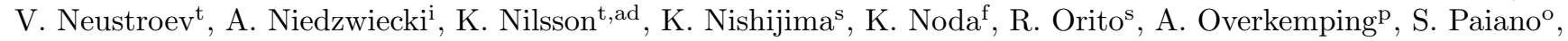

M. Palatiello ${ }^{\mathrm{b}}$, D. Paneque ${ }^{\mathrm{f}}$, R. Paoletti ${ }^{\mathrm{d}}$, J. M. Paredes ${ }^{\mathrm{r}}$, X. Paredes-Fortuny ${ }^{\mathrm{r}}$, M. Persic ${ }^{\mathrm{b}, \text { af }}$, J. Poutanen ${ }^{\mathrm{t}}$, P. G. Prada Moroni $^{\mathrm{v}}$, E. Prandinik ${ }^{\mathrm{k}, \mathrm{ag}}$, I. Puljak ${ }^{\mathrm{e}}$, R. Reinthal ${ }^{\mathrm{t}}$, W. Rhode ${ }^{\mathrm{p}}$, M. Ribór ${ }^{\text {, J. Rico }}{ }^{\mathrm{a}}$, J. Rodriguez Garcia ${ }^{\mathrm{f}}$, S. Rügamer ${ }^{\mathrm{l}}$,

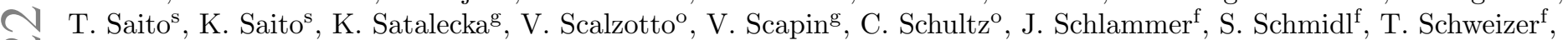
A. Sillanpäät ${ }^{\mathrm{t}}$ J. Sitarek ${ }^{\mathrm{a}}$, I. Snidaric ${ }^{\mathrm{e}}$, D. Sobczynska ${ }^{\mathrm{i}}$, F. Spanier ${ }^{1}$, A. Stamerrac ${ }^{\text {, T. Steinbring }}{ }^{\mathrm{l}}$, J. Storz ${ }^{\mathrm{l}}$, M. Strzys ${ }^{\mathrm{f}}$,

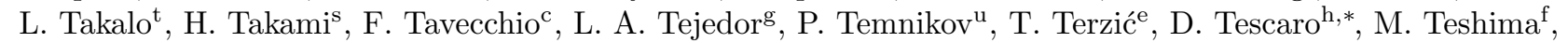

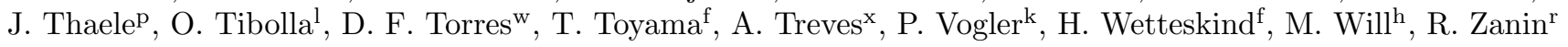

$$
\begin{aligned}
& { }^{a} \text { IFAE, Campus UAB, E-08193 Bellaterra, Spain } \\
& { }^{b} \text { Università di Udine, and INFN Trieste, I-33100 Udine, Italy } \\
& { }^{c} \text { INAF National Institute for Astrophysics, I-00136 Rome, Italy } \\
& { }^{d} \text { Università di Siena, and INFN Pisa, I-53100 Siena, Italy } \\
& { }^{e} \text { Croatian MAGIC Consortium, Rudjer Boskovic Institute, University of Rijeka and University of Split, HR-10000 Zagreb, Croatia } \\
& { }^{f} \text { Max-Planck-Institut für Physik, D-80805 München, Germany } \\
& { }^{g} \text { Universidad Complutense, E-28040 Madrid, Spain } \\
& { }^{h} \text { Inst. de Astrofísica de Canarias, E-38200 La Laguna, Tenerife, Spain } \\
& { }^{i} \text { University of Łódź, PL-90236 Lodz, Poland } \\
& { }^{j} \text { Deutsches Elektronen-Synchrotron (DESY), D-15738 Zeuthen, Germany } \\
& { }^{k} \text { ETH Zurich, CH-8093 Zurich, Switzerland } \\
& { }^{l} \text { Universität Würzburg, D-97074 Würzburg, Germany } \\
& { }^{m} \text { Centro de Investigaciones Energéticas, Medioambientales y Tecnológicas, E-28040 Madrid, Spain } \\
& { }^{n} \text { Institute of Space Sciences, E-08193 Barcelona, Spain } \\
& { }^{\circ} \text { Università di Padova and INFN, I-35131 Padova, Italy } \\
& { }^{p} \text { Technische Universität Dortmund, D-44221 Dortmund, Germany }
\end{aligned}
$$




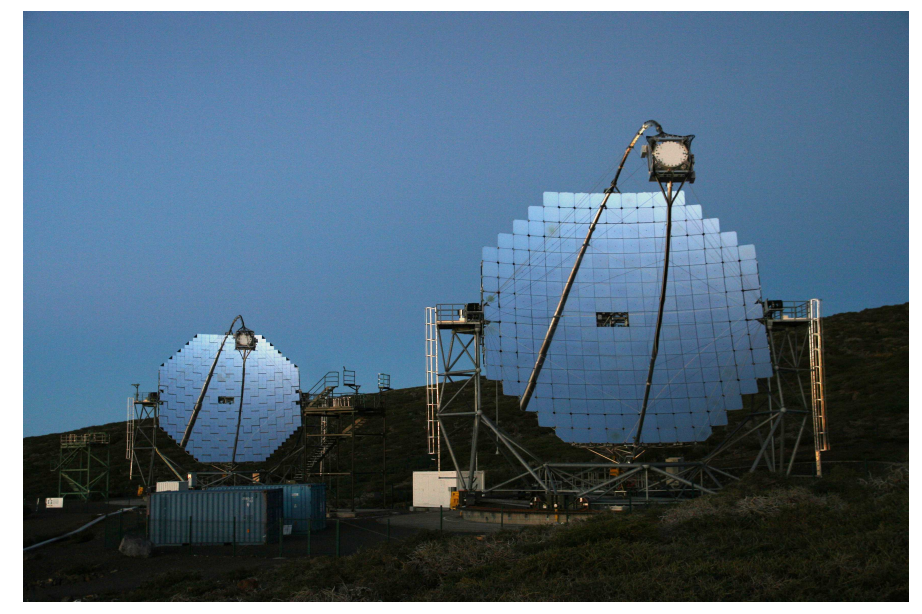

Figure 1: The two $17 \mathrm{~m}$ diameter MAGIC telescope system operating at the Roque de los Muchachos observatory in La Palma. The front telescope is MAGIC-II.

\section{Abstract}

The MAGIC telescopes are two Imaging Atmospheric Cherenkov Telescopes (IACTs) located on the Canary island of La Palma. The telescopes are designed to measure Cherenkov light from air showers initiated by gamma rays in the energy regime from around $50 \mathrm{GeV}$ to more than $50 \mathrm{TeV}$. The two telescopes were built in 2004 and 2009, respectively, with different cameras, triggers and readout systems. In the years 2011-2012 the MAGIC collaboration undertook a major upgrade to make the stereoscopic system uniform, improving its overall performance and easing its maintenance. In particular, the camera, the receivers and the trigger of the first telescope were replaced and the readout of the two telescopes was upgraded. This paper (Part I) describes the details of the upgrade as well as the basic performance parameters of MAGIC such as raw data treatment, dead time of the system, linearity in the electronic chain and sources of noise. In Part II, we describe the physics performance of the upgraded system.

\section{Introduction}

MAGIC (see Fig. 1) is a stereoscopic system of two Imaging Atmospheric Cherenkov Telescopes (IACTs) located at the observatory of Roque de los Muchachos in La Palma, Canary Islands (Spain). Together with the H.E.S.S. IACTs in Namibia (Aharonian et al. 2006) and the VERITAS IACTs in Arizona (Holder et al. 2008), MAGIC dominates the high-energy gamma-ray astrophysics in the range between between few tens of GeVs and tens of TeVs.

\footnotetext{
*Corresponding author

Email addresses: mazin@mpp.mpg.de (D. Mazin), diego.tescaro@gmail.com (D. Tescaro)
}

The two MAGIC telescopes started operation 5 years apart (2004 and 2009, respectively), and the second telescope was an "improved clone" of the first one. The main reasons for differences were economical constraints during the building of the first telescope and the technological progress that took place in the years between the design of the two telescopes. The major focus of the telescopes is a lowest possible energy threshold, which is achieved through fine pixelized cameras, fast sampling electronics and a large mirror area. The second goal is a fast repositioning speed in order to catch rapid transient events such as Gamma-Ray Bursts, which is achieved through a light weight ( $<70$ tons) telescope structure made out of reinforced carbon fibre tubes. The light weight structure requires an automatic mirror control (AMC) to maintain the best possible optical point spread function at different zenith angles of observations (Lorenz 2004, Doro 2012). The achieved energy threshold is as low as $\sim 50 \mathrm{GeV}$ at the trigger level for observations at zenith angles below $25^{\circ}$ (see Fig. 6 in Aleksić et al. 2014). The repositioning speed is maintained throughout the years to be $\sim 25 \mathrm{~s}$ for a $180^{\circ}$ rotation in azimuth.

While the above mentioned concepts made the two telescopes look very similar there were few important design changes. These were mainly motivated by the improved sensitivity of the telescopes when operating them in hardware stereo mode, i.e. requiring a coincidence trigger between the two, as well as by a better economical situation. The second telescope and the stereo trigger allowed for observations of extended sources and a better usage of the so-called wobble mode (Fomin et al. 1994) for a better background estimation. This motivated an enlarging of the trigger area in the camera and a consequent finer pixelization. In detail, the main resulting differences between the two telescopes were the following ones:

- The camera of the MAGIC-I telescope consisted of 577 pixels (divided in 397 small pixels, 1 inch diameter, in the inner part of the camera and 180 large pixels, 2 inch diameter, in the outer part). The camera of MAGIC-II consists of 1039 pixels, all small, 1 inch diameter.

- The trigger of MAGIC-II had an affective area 1.7 times larger than the one of MAGIC-I.

- MAGIC-I readout was based on an optical multiplexer and off-the-shelf FADCs (MUX-FADC, Bartko et al. 2005), which was robust and had an excellent performance but was expensive and bulky. The readout of MAGIC-II was based on the DRS2 chip ${ }^{1}$ (compact and inexpensive but performing worse in terms of intrinsic noise, dead time and linearity compared to the MUX-FADC system).

\footnotetext{
${ }^{1}$ See http://drs.web.psi.ch/
} 
- The receiver boards of MAGIC-I (see Sec. 3.3.1) lacked of programmability. They were also showing high failure rate, mainly due to ageing.

In 2011-2012 MAGIC underwent a major upgrade program to improve and to unify the stereoscopic system of the two telescopes. Most importantly, the camera of MAGIC-I was replaced by a new one, the readout of the two telescopes replaced by a more modern system, and the trigger area of the MAGIC-I was increased to match the one of the MAGIC-II. This paper (Part I) describes the motivation of the upgrade, its main steps, the commissioning of the system and the low level performance of MAGIC. In Part II (Aleksić et al. 2014) we describe the physics performance of the upgraded system.

\section{Motivation of the upgrade}

There were three main motivations of the upgrade of the MAGIC system. The first one was the wish to improve the stereoscopic performance of the MAGIC system. Several key parameters were targeted for improvement:

- The low energy performance. The performance of MAGIC to the lowest accessible energies was limited by the electronic noise in the DRS2 system of the MAGIC-II telescope. With a lower noise system the analysis energy threshold can be lowered, and the performance close to the threshold can be improved.

- The flux sensitivity to extended sources. The small trigger area of the MAGIC-I telescope (1 degree diameter) was hindering a study of extended Galactic gamma-ray sources, with angular sizes $\geq 0.3^{\circ}$. A $70 \%$ larger trigger area, the same as in the MAGIC-II telescope, allows to simultaneously measure an extended source up to $\sim 0.5^{\circ}$ extension, and a better control of the background region.

- The dead time of the system. Due to the intrinsic constraints of the DRS2 based readout of MAGICII, the dead time of the system was $500 \mu$ s for every recorded event, which was translating into a $\sim 12 \%$ dead time. Reducing the dead time per event by a factor of $\sim 10$ was one of the goals of the upgrade in order to effectively gain $\sim 12 \%$ of the observation time.

- The angular resolution for gamma rays. Replacing the MAGIC-I camera with one containing small pixels only, the image parameters can be better determined, which helps in the reconstruction of the primary gamma-ray characteristics such as their incoming direction.

The second main motivation was an improvement of the operation stability and a significant reduction of any downtime due to technical problems. The main target was to replace or upgrade sub-systems that had given technical problems during the first years of MAGIC operation. Moreover, many diagnostic and monitoring online tools have been developed and installed to immediately alert the shifters and subsystem experts in case of any malfunctioning. A special attention was given to producing and storing in La Palma a sufficient amount of spares for most of the hardware.

The third motivation was the unification of the MAGICI and MAGIC-II telescope hardware to reduce maintenance costs and ease operation. The goal was to reduce the amount of expert manpower to secure a stable MAGIC operation for the following years.

\section{Individual parts of the upgrade}

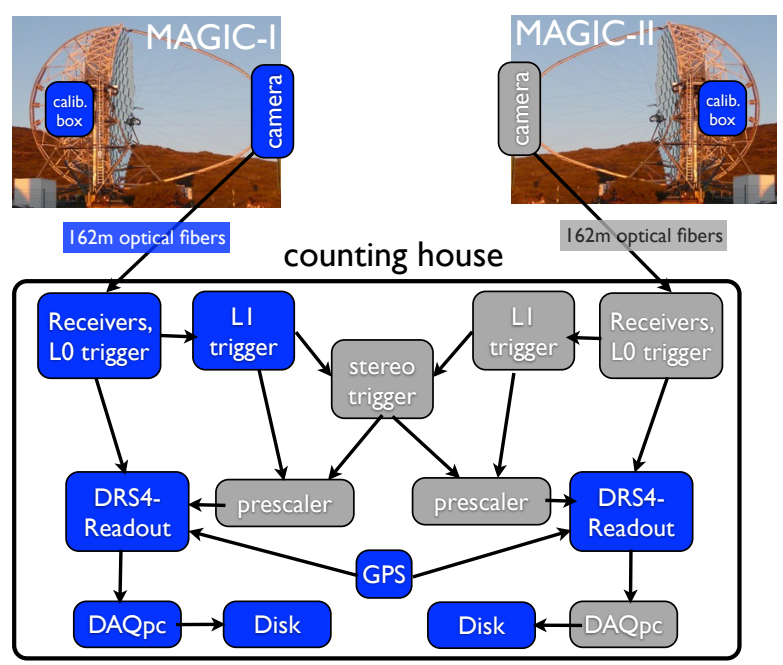

Figure 2: Schematic view of the readout and trigger chain of the MAGIC telescopes. The blocks in the blue boxes have been replaced and commissioned during the upgrade.

In this section we describe the main hardware parts that have been upgraded. The individual hardware items of the upgrade program are shown in Fig. 2 .

\subsection{Camera of the MAGIC-I telescope}

The new MAGIC-I camera has 1039 channels and follows closely the design and the performance of the MAGIC-II camera (Borla-Tridon et al. 2009). The photosensors are photomultiplier tubes (PMTs) from Hamamatsu, type R10408, 1 inch diameter, with a hemispherical photocathode and 6 dynodes. Each pixel module includes a compact power unit providing the bias voltages for the PMT and a stack of round circuit boards for the frontend analog signal processing, see the configuration in the upper photo of Fig. 3. The PMT bias voltages for the cathode and dynodes are generated by a low power, nine step Cockroft-Walton DC-DC converter, which can provide up to $1250 \mathrm{~V}$ peak voltage. We operate the 6 dynodes 


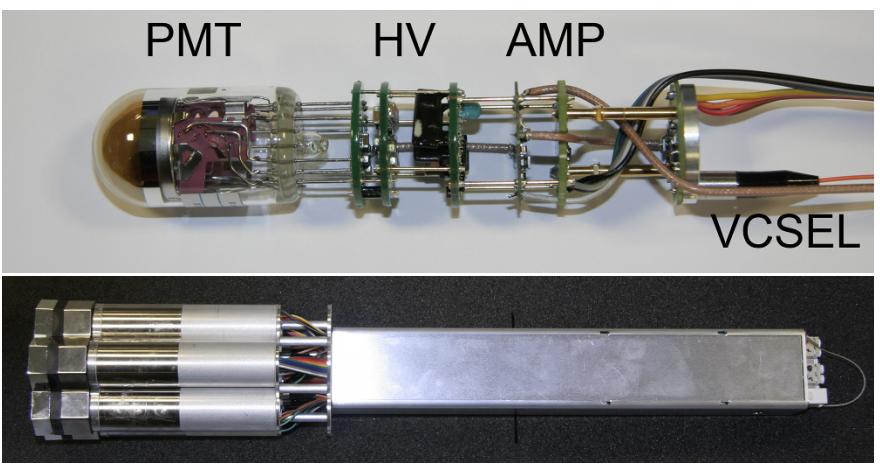

Figure 3: Assembled PMT module to form a pixel in the upper image and a full cluster of 7 pixels in the bottom image.

PMTs at a rather low gain of typically $3-4 \cdot 10^{4}$ (see below) in order to also allow observations under moderate moonlight without damaging the dynodes. The pixels are grouped in 7 to form a cluster for an easier maintenance (lower picture in Fig. 3). An electrical signal (called pulse injection) can be injected at the PMT base of every pixel allowing for day-tests of the whole electrical chain from the PMT base down to the readout and trigger without applying a high voltage to the PMTs. The pulse injection signals have similar shape as the Cherenkov light pulses (FWHM of $2.6 \mathrm{~ns}$ ) to have a realistic system response.

The main difference to the MAGIC-II camera pixels is the PMT gain. The mean gain of the MAGIC-II PMTs is about $3.0 \times 10^{4}$ at $850 \mathrm{~V}$ whereas half of the MAGIC-I PMTs have a gain of $3.0 \times 10^{4}$ and, for the same PMT voltage, other half a higher gain of $4.5 \times 10^{4}$. The reason for ordering higher gain PMTs for the MAGIC-I camera was the intrinsic spread in the gains observed in the MAGIC-II PMTs and a wish to counter act ageing effects. The intrinsic spread of the gains leads to the need of the high voltage (HV) flatfielding procedure (see Section 5.2 resulting in a significant spread of applied HVs. Having ordered PMTs with two different gains allowed to better sort the PMTs according to their actual gain into high-gain and low-gain PMTs, making a cut at a nominal value of $3.0 \times 10^{4}$. The analog signals of the high-gain PMTs are then attenuated in the PMT clusters by a a factor of two (using a resistor), resulting in a narrow range of applied HVs in the camera after the $\mathrm{HV}$ flatfielding procedure. It is foreseen to remove the resistor in the high-gain PMTs once the ageing effect considerably lower their gain.

\subsection{Optical cables}

The optical cables continuously transmit analog signals from the PMTs to the readout and trigger electronics located in the control house. The optical fibers are $\sim 162 \mathrm{~m}$ long and are grouped in 19 bundles (per telescope) for a better handling, 72 fibers each, allowing for sufficient amount of spare fibers in case some break. The bundles

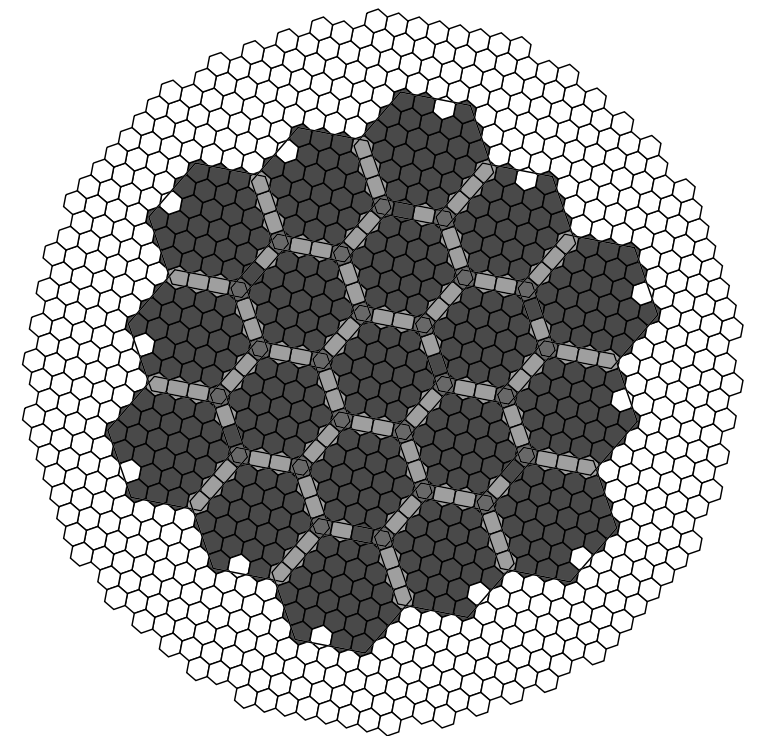

Figure 4: Geometry of the MAGIC cameras with 1039 channels, each. The dark grey hexagons (36 pixels each) show the 19 L1 trigger macrocells. Pixels that are covered by more than one macrocell are shown in light grey.

are protected by a UV resistant PVC cover to ensure mechanical rigidity, protect the fibers from breaking and from the strong sun UV radiation in La Palma. It is important to prevent divergence of arrival times between individual channels due to different times of flight in the optical fibers. Therefore, a special setup was developed to manufacture and control that the propagation time is uniform in the fibers. The resulting spread in the propagation time is $138 \mathrm{ps}$ (RMS), and maximum difference of $650 \mathrm{ps}$. This spread is corrected for offline using calibration light pulses.

\subsection{DRS4 based readout}

The DRS4 based readout system is the major technical novelty of the upgrade. DRS4 stands for Domino Ring Sampler version 4, to distinguish it from its predecessor DRS2. The new readout sampling the signals with 2 Gsamples/s is cost effective, has a linear behavior over a large dynamic range (from less than 1 photoelectron (phe) to about 600 phe), less than $1 \%$ dead time, low noise, and negligible channel-to-channel cross-talk (Sitarek et al. 2013 Bitossi et al. 2014). This allowed us to maintain the performance of the previous readout based on MUXFADCs while increasing the charge resolution, reducing 
cost and saving space. The space argument is not circumstantial. In fact, the electronics room hosting the trigger and readout of the two telescopes was not large enough to host a readout of more than 2000 channels in a previous configuration. Through the upgrade to a more compact DRS4 system (96 readout channels per $9 \mathrm{U}$ board), only 6 racks are needed for the trigger and readout system of the two telescopes (see Fig. 6 and 7).

The baseline concept of the readout system, now adopted in both telescopes, is the same as the one used in MAGIC-II in 2009 (Tescaro et al. 2009). The readout electronics is divided in two main parts: the receiver boards and the digitization electronics, both controlled by the same VME-based communication network 2

\subsubsection{Receiver boards}

PMT signals are split in the Magic Optical Nano-Second Trigger and Event Receiver (MONSTER boards or receiver boards in short) into analog - readout and sumtrigger, see below - and digital branches. The optical fibers, carrying the optical PMTs signal to the control house, connect on the back side of the MONSTER boards by means of LX5-LX5 optical connectors. The MONSTER is a multilayer $9 \mathrm{U}$ board with the following tasks:

- convert optical signals from the camera back to analog electrical ones.

- bring analog signals to the digitization electronics.

- generate the Level-0 (L0) individual pixel trigger signal using discriminators.

- further split the analog branch in order to feed a copy of the signals to the analog trigger (sum-trigger, Rodriguez Garcia et al. (2013)).

In the analog branch, the optical receivers have a bandwidth of $800 \mathrm{MHz}$, a gain of $18.5 \mathrm{~dB}$, a negligible cross-talk of $0.1 \%$ and a working range from $0.25 \mathrm{mV}$ (corresponding to $\sim 0.15$ phe) to $1150 \mathrm{mV}$, with an RMS noise smaller than $0.2 \mathrm{mV}$. A single board holds 24 channels with a maximum power consumption of $75 \mathrm{~W}$.

Three parameters of the L0 trigger can be adjusted from a PC via VME for each individual channel: (1) the discriminator thresholds (DT), (2) the delay, and (3) the width of the output pulse of the discriminators. The thresholds and the delays/widths can be adjusted with a precision of $0.07 \mathrm{mV}(\sim 0.04 \mathrm{phe})$, and $10 \mathrm{ps}$, respectively. The individual pixel rate (IPR) can be monitored at a rate up to $1 \mathrm{kHz}$ but is currently monitored at $1 \mathrm{~Hz}$, which is sufficient for a reaction to stars in different fields of view (see Section 5.3.4.

\footnotetext{
${ }^{2}$ CAEN-CONet daisy-chain network (using the CAEN A2818 PCI-card and the CAEN V2718 optical linked VME bridges).
}

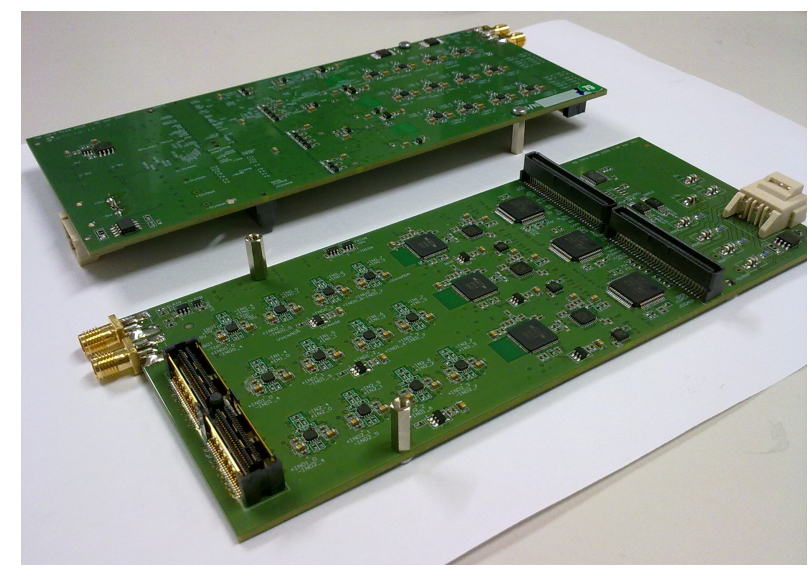

Figure 5: Picture of the DRS4 mezzanine developed at the INFN/Pisa electronics laboratories. From left to right one can recognize the two SMA connectors for the external synchronization signals (trigger and reference clock), the analog connector, the operational amplifiers to drive the input signal to the DRS4 chips, the three DRS4 chips (using 8 digitization channels each, 24 channels in total per mezzanine), the three built-in FIFO memories, the connector to interface the host motherboard and the external power supply connector.

\subsubsection{Digitization electronics}

The sampling electronics is built with a motherboardmezzanine logic, where the motherboard is the PULSAR board designed at the University of Chicagd ${ }^{3}$, and the mezzanine is the new DRS4 mezzanine (Fig. 5) designed at the INFN/Pisa laboratory (Bitossi et al. 2014). As mentioned above, the new DRS4 mezzanine uses now the DRS4 chip instead of the DRS2 chip adopted in 2009 for MAGIC-II. Conceptually, it is an ultra-fast analog memory (a ring buffer built of 1024 switching capacitors) that is read out - only in the event of a trigger - at a lower speed by a conventional analog to digital converter. In our case we use a 14-bit nominal resolution analog to digital converters (ADC), clocked at $32 \mathrm{MHz}$. The DRS4 chips has a built-in Region of Interest (RoI) selection mode that reduces drastically the time overhead for the readout of the chip. The dead time now is of $27 \mu$ s only (negligible in standard data acquisition conditions). The DRS4 chip has tuneable sampling frequency (from 700 Msamples/s to 5 Gsamples/s) set to 2 Gsamples/s and linear response in an input range of $1 \mathrm{~V}$. The mezzanine noise is $\sim 7.5 \mathrm{ADC}$ counts, corresponding to $\sim 450 \mu \mathrm{V}$ at the board input, and is dominated by the noise from the DRS4 chip which varies widely from chip to chip (Bitossi et al. 2014). The measured bandwidth is $\sim 650 \mathrm{MHz}$. Overall, the digitization electronics contribute to $\sim 50 \%$ of the total noise (see Section 4.1.

A total of 48 DRS4 mezzanines are installed in each readout, for a total of 1152 readable channels (enough to

\footnotetext{
${ }^{3}$ http://hep.uchicago.edu/thliu/projects/Pulsar/
} 


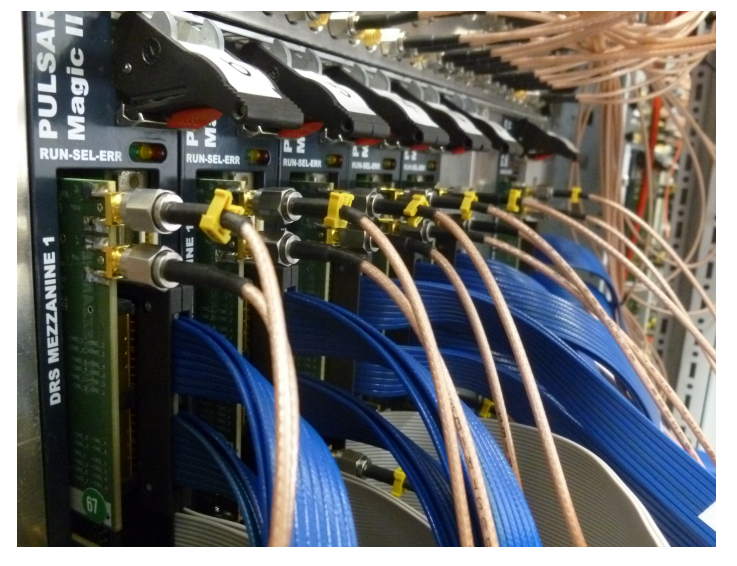

Figure 6: A close-up into the electronics, which read out the ultrafast signals produced by the Cherenkov photons from cosmic-ray induced atmospheric showers in the camera of the MAGIC telescopes. The DRS4 mezzanines (the green cards hosted by the PULSAR motherboards) are connected to the receiver boards by means of 24differential-lines analog cables (blue) and synchronized by two SMA cables (one for the trigger signal and one for the common reference clock signal).

cover the 1039 camera pixels and keep $\sim 10 \%$ spare channels). A close-up of several PULSAR boards holding the DRS4 mezzanines is shown in Fig. 6.

The final data acquisition (DAQ) is performed in a single computer per telescope steered by a multithread $\mathrm{C}++$ program (Tescaro et al. 2013). The readout electronics communicates with the DAQ via the SLink optical data transfer system, with the HOLA cards ${ }^{4}$ on the readout side and the FILAR PCI cards $\$^{5}$ on the computer side.

\subsubsection{Readout data pre-processing}

The calibration of the chip response is mandatory to obtain optimal results in terms of noise and time resolution (see Sitarek et al. 2013). Three important corrections are applied to the data:

- The mean cell offset calibration

- The readout time lapse correction

- The signal arrival time calibration

Currently the first two are applied online by the DAQ program whereas the third is applied offline (although all the corrections can be applied offline if required).

Fig. 8 shows the mean cell offset (and its RMS) as a function of the absolute position of the capacitor (cell units) in the DRS4 ring buffer for a typical channel. Notice that

\footnotetext{
${ }^{4}$ see: https://hsi.web.cern.ch/hsi/s-link/devices/hola/

${ }^{5}$ see:

link/devices/filar/welcome.html
}

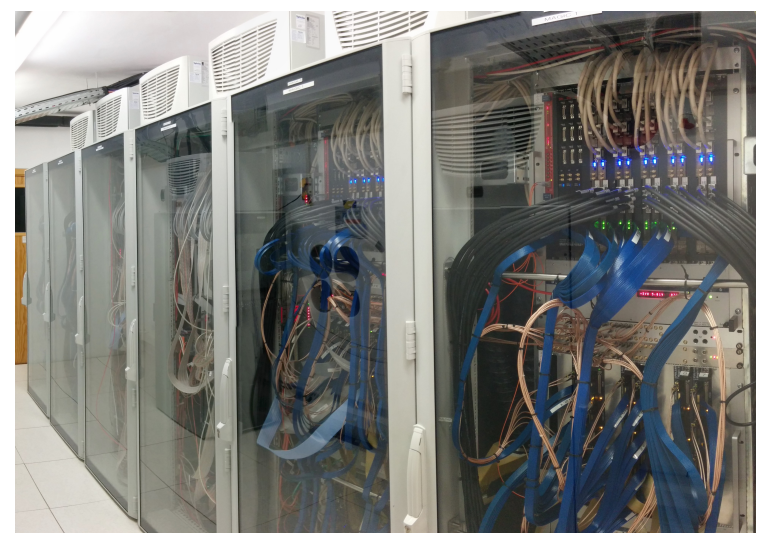

Figure 7: View of the electronics room of the MAGIC telescopes. The six closed racks can be seen. They are placed on a technical raised floor $(20 \mathrm{~cm}$ height $)$ allowing for better cable routing.

the single capacitor baseline varies greatly from cell to cell, well beyond the noise fluctuations. To equalize the response and obtain a flat baseline the mean cell offset of each cell is computed using a dedicated DRS4 pedestal calibration run, and subtracted to the readout values. This is what we call the mean cell offset calibration of the chip and has to be done with a special algorithm that takes into account not only the absolute capacitor position in the buffer but also the trigger position in the ring (see Sitarek et al. 2013).

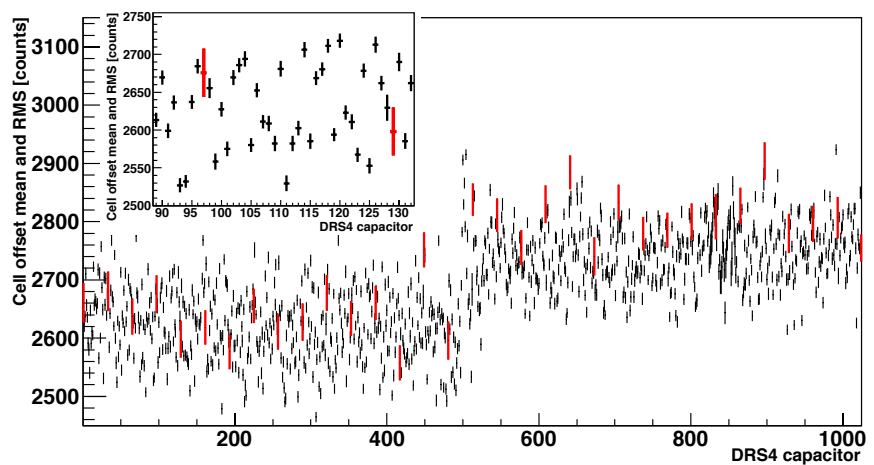

Figure 8: Cell offset of 1024 individual capacitors of one channel of the DRS4 chip. Vertical error bars show the standard deviations of the offset values for the capacitors. Every $32^{\text {nd }}$ capacitor is marked with a thick red line. The inside panel zooms into some of the capacitors to better appreciate the differences from capacitor to capacitor (Sitarek et al. 2013).

The mean cell offset calibration has to be further corrected since the mean offset suffers a dependency with the last reading time lapse: the offset decreases following a simple power law as a function of the time passed. Since this behavior is very similar for all the DRS4 chips, a uni- 
versal analytical expression can be used to further correct the single capacitor's offsets. If not corrected, this effect would produce steps in the baselines (see Fig. 9, , since for a given readout of the chip only a small part of the buffer is actually readout.
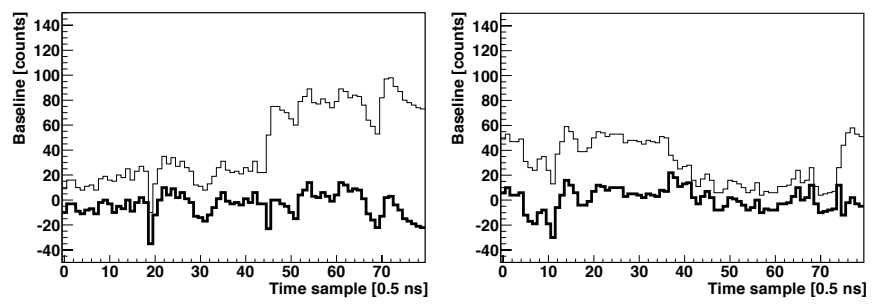

Figure 9: Examples of digitized pedestal data with the DRS4 chip. The capacitor offset depends on the time lapse with the last readout of the capacitor, which results in steps on the baseline in case of irregular triggers. The thin line shows the original data and the thick line show the effect of the time lapse correction.

Finally, the DRS4 exhibits a moderately variable time spread (1-4 ns) on the delay of the recorded signal pulses, depending on the absolute position in the ring buffer (see Fig. 10. This effect is highly chip-dependent and has to be calibrated independently for each DRS4. The characteristic delay figures are built by means of calibration runs (synchronous pulses of fixed amplitude) and parameterized using Fourier series expansions. This basic arrival time calibration recovers the true arrival time at the DRS4 input, resulting in a characteristic time spread of $\sim 0.2 \mathrm{~ns}$ (Sitarek et al. 2013).

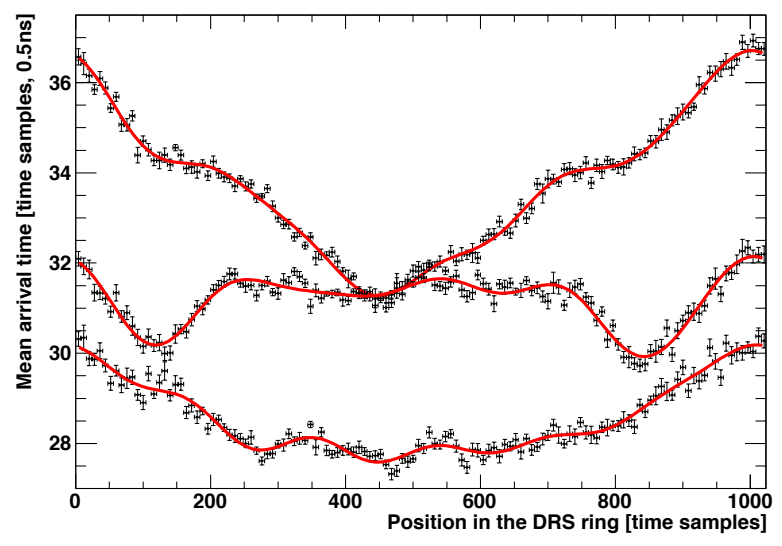

Figure 10: Mean pulse signal arrival time as a function of the position in the DRS4 chip run buffer for three typical channels together with their Fourier series expansion (solid lines).

\subsection{Individual telescope trigger and stereo trigger}

In the MAGIC-II and the upgraded MAGIC-I cameras, the trigger region covers the 547 inner pixels. The MAGIC trigger has three levels. The first trigger level (L0) is a simple amplitude discriminator operating on each pixel individually. For each telescope, the 547 digital L0 signals generated by the receiver boards (see Section 3.3.1) are sent to the second trigger level, the telescope trigger (L1). The width and the delay of the digital L0 signals are set individually, which is important to minimize the spread of arrival times for contemporaneous signals for the L1 trigger (see below).

The L1 trigger is a digital filter arranged in 19 macrocells of 36 channels each, with a partial overlap of channels between the macrocells (see Fig. 4). Several logic patterns are implemented: 2 next-neighbor logic $(2 \mathrm{NN}), 3 \mathrm{NN}, 4 \mathrm{NN}$ and $5 \mathrm{NN}$. The patterns are always close compact. In case any of the 19 macrocells reports a coincidence trigger of the programmed logic, a L1 trigger signal (also called individual telescope trigger) is issued. The upgraded trigger has the same number of macrocells as the previous one but the overlap between them was reduced from three pixel rows to one. The smaller overlap created $\sim 1 \%$ trigger inefficiency for $3 \mathrm{NN}$ and $4 \mathrm{NN}$ logic but increased the trigger area by a factor of $\sim 1.7$ as stated above.

The L1 trigger signals are sent to the third trigger level, the stereo trigger (L3). The L1 signals are artificially stretched to $100 \mathrm{~ns}$ width and are delayed according to the zenith and azimuth orientation of the MAGIC telescopes to take into account the differences in the arrival times of the Cherenkov light from air showers. A simple coincidence between the two signals is made and the resulting signal (L3 output) is sent back to the individual telescope readout. The width of $100 \mathrm{~ns}$ for the two signals is chosen to ensure a safe margin for a $100 \%$ L3 efficiency even in case of some misalignment in the timing between the two telescopes. The L3 coincidence has a intrinsic jitter of about \pm 10 ns due to the angular difference between the shower axis of the triggered events and the pointing direction of the telescopes. We describe performance parameters of the trigger in the commissioning section below.

\subsection{Calibration system}

The calibration of the MAGIC telescopes is performed through the uniform illumination of the PMT camera with well-characterized light pulses of different intensity produced by a system, which we name calibration box, installed at the (approximate) center of the mirror dish, i.e. about $17 \mathrm{~m}$ away from the camera plane. The MAGICI calibration box was installed in 2004, and was based on fast-emitting (3-4 ns FWHM) LEDs (Schweizer et al. 2002). The light intensity was adjusted by changing the number of LEDs that fired, and the uniformity was achieved by a diffusor at the exit window. On the other hand, the MAGIC-II calibration box (installed in 2009) is based on a system with a passively Q-switched Nd:YAG laser (third harmonics, wavelength of $355 \mathrm{~nm}$ ) that produces pulses of $0.4 \mathrm{~ns}$ FWHM. The light intensity is adjusted through the selection of a calibrated optical filter and the uniformity is achieved by means of an Ulbricht 
sphere that diffuses the light right before the exit window. After the Ulbricht sphere the laser pulse has a FWHM of $\sim 1 \mathrm{~ns}$, which is similar to the time spread of the photons in the Cherenkov shower.

The laser-based system was proven superior to the LEDbased system because it provides (a) a larger dynamic range, and (b) shorter light pulses ( $<2 \mathrm{~ns}$ FWHM), which are more similar to the ones produced by the Cherenkov flashes from extended air showers.

For the upgraded MAGIC system (both MAGIC-I and MAGIC-II) we decided to use a calibration box similar to that originally installed in MAGIC-II but with some performance upgrades: (a) a humidity sensor inside the box, (b) the laser status can now be queried, (c) a heating system attached to the entrance window to avoid water condensation, (d) a fast photodiode for monitoring the laser light output, (e) an improved dynamic range, together with a more detailed characterization of the light intensities, and $(f)$ an improved uniformity in the illumination of the telescope camera with variations of less than $<2 \%$.

Before each observation, a calibration run consisting of 2000 events at a fixed light intensity is taken. The extracted charge per pixel and its variance are used to determine the conversion factor between the ADC counts of the readout and the number of phe via the $\mathrm{F}$-factor method, that relies on the knowledge of the added noise of the PMT (Mirzoyan and Lorenz 1997). The calibration light pulses are also used to cross-calibrate the analog arrival times in the DRS4 channels, which are different channel by channel (due to differences in propagation time between the focal plane and the DRS4 chip) and depend on the position of trigger signal in the DRS4 ring buffer (see Sitarek et al. 2013). In addition, during data taking the calibration laser is constantly firing at $25 \mathrm{~Hz}$ (so-called interleaved calibration events) allowing the monitoring of the gain in the readout chain of the individual channels.

\subsection{Computing}

The computing infrastructure of the MAGIC telescopes was also upgraded as a part of the general hardware upgrade.

Most of the computing equipment was moved from the electronics room to an adjacent, newly prepared dedicated computer room. Four racks containing computers, storage elements and network equipment were installed in the new location. All equipment was connected to power switches that could be controlled remotely. New computers were also added to the cluster of analysis machines to process data on-site and the volume of the storage elements was doubled by using new disks in this disk matrix. More details on the storage area configuration can be found in Carmona et al. (2009).

A major upgrade of the operating system became necessary since it was not possible to keep old operating system for newer computers. The computers are split into a cluster of the on-site analysis machines, susbsystem machines (needed for operation of the telescopes) and the storage area network (SAN). The analysis computers that could access the SAN were updated to a new operating system version (Scientific Linux CERN 6.3) and new computers were added. Two storage elements (RAID 1 and RAID 2 ), where the raw data is written by the DAQ machines, were separated from the rest of the $\mathrm{GFS}^{6}$ cluster and formatted as XF\$7, see Fig. 11. The raw data is copied to RAID 3 (MAGIC-I data) and RAID 4 (MAGIC-II data) during the data taking. The raw compressed files are already available for full analysis on the RAID 3 and RAID 4 partitions few minutes after the end of the observations, which allows the on-site analysis machines to start processing them timely. The capacity in each of the two storage units, that are connected to the DAQ machines directly, is 7.3 TBytes for the main partition and 3.7 TBytes for the backup partition. The volume in the main partition of this elements is large enough to contain more than 5 full nights of uncompressed data under normal operation conditions. The total capacity of the storage units is $\sim 100 \mathrm{~TB}$.

High level analysis results are produced on-site by all analysis nodes using the standard analysis software MARS (Zanin et al. 2013), and are usually available a few hours after the end of the data taking. In this way, the on-site analysis does not interfere with the data taking and raw file writing procedure. These processed data files and the original raw data files are copied to the MAGIC datacenter at PIC, Barcelona, Spain (Reichardt et al. 2009), where they are permanently stored and made available to the whole collaboration. The copying of the data to PIC is done from the Grid node of the GFS2 8 cluster using efficient Grid tools for data transfer of large data volumes. Fig. 11 shows the schematic view of the configuration for DAQ machines and GFS2 cluster after the changes introduced in mid 2012. The configuration of the system is however very flexible and it allows to add easily new computers or new storage elements.

\subsection{The timing system}

The previous timing system consisted of several separate units: a Rubidium clock (Rubclock), a GPS module and several NIM modules. The timing information were converted to LVDS format and fed to the readout at the time of the trigger in order to timestamp events, so the 44 bit LVDS signal required 88 physical cables divided into four connectors (three 40 pin connector and one 16 pin connector). The precision of the system was $200 \mathrm{~ns}$. Since the system became difficult to maintain (several modules were in use since the HEGRA experiment in the 90's, Daum et al. (1997)) it was decided to build a new timing system.

\footnotetext{
${ }^{6}$ Global File System for a shared disk file systems for Linux computer clusters

${ }^{7} \mathrm{XFS}$ is a high-performance 64 -bit journaling file system.

8 GFS2 is a further development derived from GFS and was included along with its distributed lock manager (shared with GFS) in Linux 2.6.19
} 


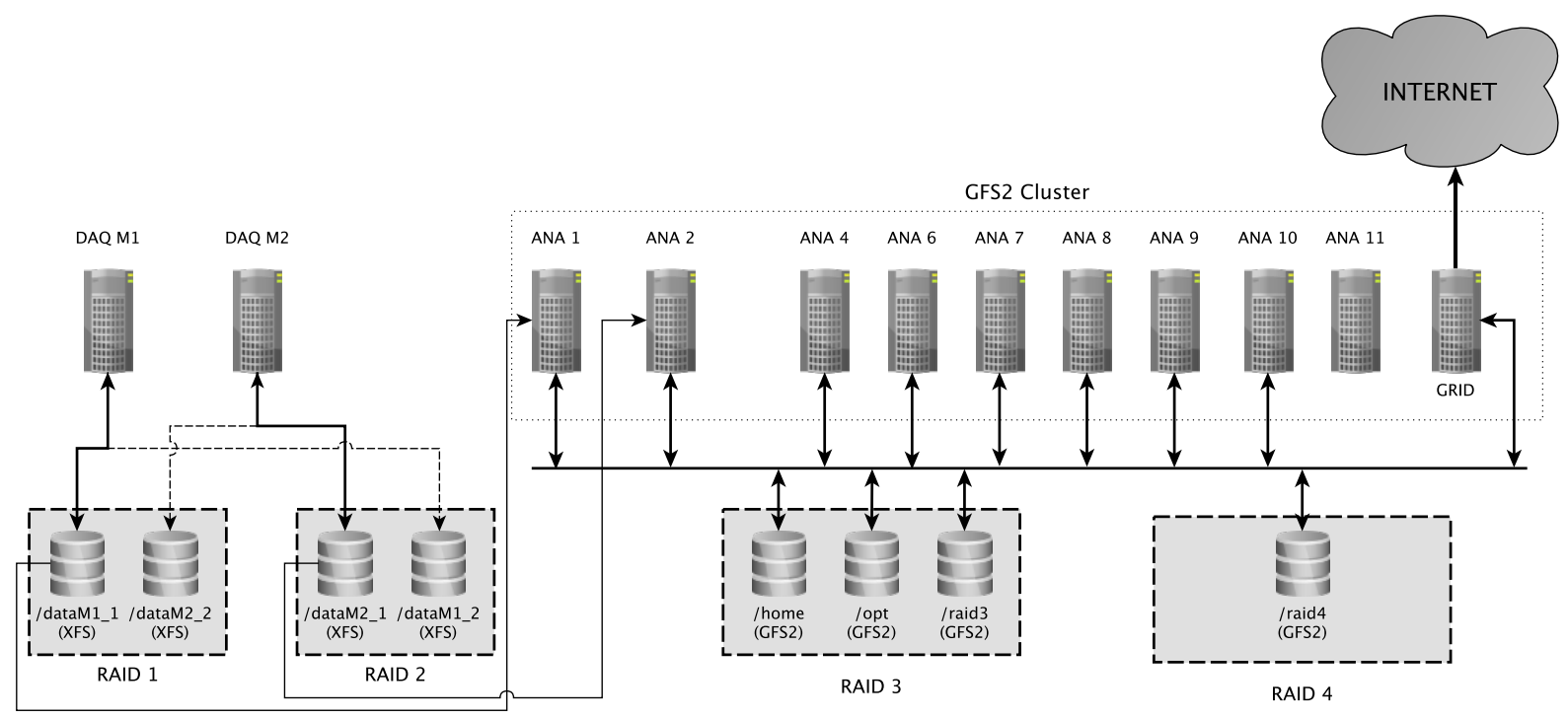

Figure 11: Schematic view on the computing network of the DAQ and the data analysis machines in MAGIC.

The Rubclock and GPS modules were substituted by a single commercial timing system ${ }^{9}$. The system is coupled to a custom-built timing rack module, which contains all the electronics needed to export valid timing information in LVDS format for both MAGIC-I and MAGIC-II. The precision of the new system is the same as for the old one: $200 \mathrm{~ns}$. However, in the old system there was a drift between the UTC 1 pulse per second (1PPS) and the 1PPS signal from the Rubidium clock, which could exceed $1.5 \mu \mathrm{s}$ and had to be reset manually, typically once a month. The drift is reduced to $65 \mathrm{~ns}$ in the new system and does not need a manual reset, which makes the system more accurate.

\subsection{The central control program}

A central control program running on a computer allows the telescope operators to perform and monitor observations (Giavitto 2013). It allows to configure and control all subsystems of the telescopes. It provides a unified graphical user interface, which allows to easily execute many complex operations. Tasks that require the synchronization of many subsystems are coded as modular routines, which can be called individually by the user. All subsystem configuration parameters are kept in plain text configuration files. This architecture allows for great flexibility and rapid development cycles. During the upgrade, the existing routines and configuration files were adjusted to the newly introduced subsystems. In some cases, the changes introduced by the upgrade allowed for further automation of some tasks, so the corresponding routines had

\footnotetext{
${ }^{9}$ http://www.symmetricom.com/products/gps-solutions/gpstime-frequency-receivers/XLi/ (Symmetricom XLi).
}

to be coded anew. A real time monitor of the data readout was also written, allowing experts to inspect every channel down to the sample level. Many new features were included during the upgrade, these include: automatic startup and shutdown procedures, an online monitor and long-term database of critical parameters of the telescope (e.g., temperatures, rates etc.), and an automatic Gamma-Ray Burst procedure not requiring human intervention ${ }^{10}$ Furthermore, an automatic procedure has been established to routinely take images of stars for monitoring the telescope tracking accuracy, pointing precision and optical PSF.

\section{Low level performance}

Here we shortly describe the basic performance parameters of the MAGIC telescope system after the upgrade.

\subsection{Sources of noise}

The two main sources of noise in the extracted signals are electronic noise and fluctuations of the night sky background (NSB). The goal of the upgrade was to keep the electronic noise at a similar level as the noise coming from the extragalactic (dark time, no bright stars) NSB. The individual contributions of the noise were extracted by dedicated runs taken with certain contributions on and off separately. First only readout electronics was switched on allowing to measure the contribution from the DRS4 and the receivers. Then the bias current of the camera VCSELs

\footnotetext{
10 Gamma-Ray Bursts are transient events of very short duration (seconds) and in order to increase chances to catch them with Cherenkov telescopes reaction time must be minimized. Therefore, automatic procedures not requiring human intervention are essential.
} 


\begin{tabular}{c|c|c} 
Source & MAGIC-I & MAGIC-II \\
\hline \hline DRS4+receivers & 0.76 phe & 0.69 phe \\
\hline VCSEL & 0.30 phe & 0.30 phe \\
\hline NSB (extragalactic) & 0.60 phe & 0.72 phe \\
\hline \hline total & 1.0 phe & 1.0 phe
\end{tabular}

Table 1: Contribution to noise from different hardware components as well as from the NSB for MAGIC-I pixels and MAGIC-II.

(vertical cavity laser diodes, see also in Borla-Tridon et al. (2009)) was turned on, and finally the HV was applied to the PMTs and camera opened during night pointing to a dark patch of the sky. The assumption in determining the individual components of the electronic noise is that they are mainly independent of each other. The obtained numbers are summarized in Table 1. One can see that the electronics noise (RMS) from the readout is at the level of 0.7 phe, the contribution from the camera (mainly VCSEL for the optical signal transmission) of 0.3 phe, which is to be compared with the level of the NSB of $0.6-0.7$ phe. Note that the level of the electronics noise in phe depends on the target HV used in the flatfielding procedure (Section 5.2). The applied HVs to the PMTs do not contribute to the noise in any measurable way. The measured NSB level is higher in MAGIC-II because of newer mirrors that have a higher absolute reflectivity than the MAGIC-I mirrors (Doro et al. 2008). The relative precision of the measurements is at the level of a few per cent. The absolute scale of the measurement is about $10 \%$, mainly due to the uncertainties procedure from $\mathrm{ADC}$ counts into phe.

\subsection{Linearity in the signal chain}

For the linearity of the readout chain we refer to a more detailed study in Sitarek et al. (2013). The linearity of the full electronics chain (PMT to the DRS4 readout) is better than $10 \%$ deviation in the range from 1-2 phe (though it is very difficult to measure 1 phe signals since the noise level is of the same order of magnitude) to few hundred phe (see Fig. 12). Some non-linearity of the order of $10-20 \%$ is observed for pulses with charge between 200 phe and 1000 phe, and signals saturate the readout (at the stage of the receiver board) at $>1000$ phe. The non-linearity effect at high charges is mainly due to the behavior of the VCSELs. Simulations showed that a non-linearity of that magnitude does not affect image parameters of events with a charge lower than 10,000 phe and has a 1-3\% effect for events with a higher charge.

\section{Commissioning of the system}

The commissioning of the upgraded system required a dedicated, well experienced and highly motivated team of 5 to 10 physicists to stay at the site of the experiment for a duration of several months after the installation of

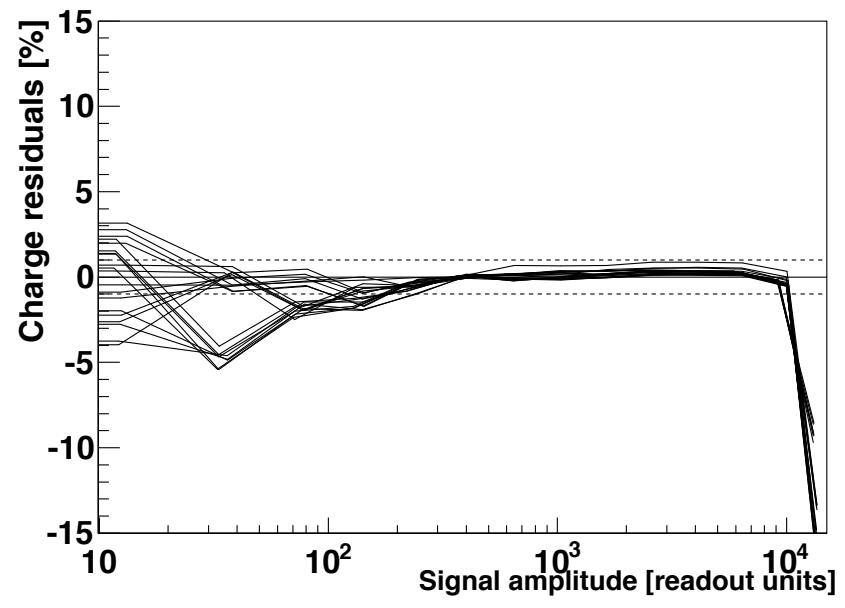

Figure 12: Deviation from linearity for 20 typical channels of the DRS4 readout (Sitarek et al. 2013). The dashed lines mark 1\% deviation. A single photoelectron has an amplitude of $\sim 30$ readout counts.

the hardware. In the following the main milestones of the commissioning are described.

\subsection{Optical point spread function}

The optical point spread function (PSF) was improved during the upgrade. A dedicated active mirror control (AMC) hardware and software (Biland et al. 2008) takes care of mirror adjustment depending of the zenith angle of observation due to small deformations of the telescope dish. After the new MAGIC-I camera was installed, counterweights on the back side of the structure had to be modified in order to compensate for the heavier weight of the new camera. Once works on the camera and the counterweights were finished, a new set of look up tables (LUTs) for the AMC were produced to achieve minimal optical PSF at every telescope pointing direction. The LUTs were produced by pointing the telescopes to stars at different zenith angles and minimizing the optical PSF (calculated from the reflected image of the star) by moving the actuators of the mirror panels. Images of stars are taken regularly by a special high sensitivity CCD camera $\left(\mathrm{SBIG}^{\mathrm{R}}\right)$ ) located in the center of the dish. A typical image defining the optical PSF for both telescopes is shown in Fig. 13 , where the $39 \%$ light containment radius is 1.86 ' $\left(1.80^{\prime}\right)$ and $95 \%$ containment radius is $7.46^{\prime}\left(6.51^{\prime}\right)$ for the MAGIC-I (MAGIC-II) telescope, respectively. Note that the MAGIC camera pixel size has a dimension of $30 \mathrm{~mm}$ (flat-to-flat of the hexagonal entrance window of the Winston cone) corresponding to a field of view of 6 '. The stability of the PSF and the absolute reflectivity of the mirrors is the subject of a forthcoming publication.

\subsection{High voltage flatfielding}

Each PMT has a different gain at a fixed HV. The spread of the gains is unavoidable during the manufacturing pro- 

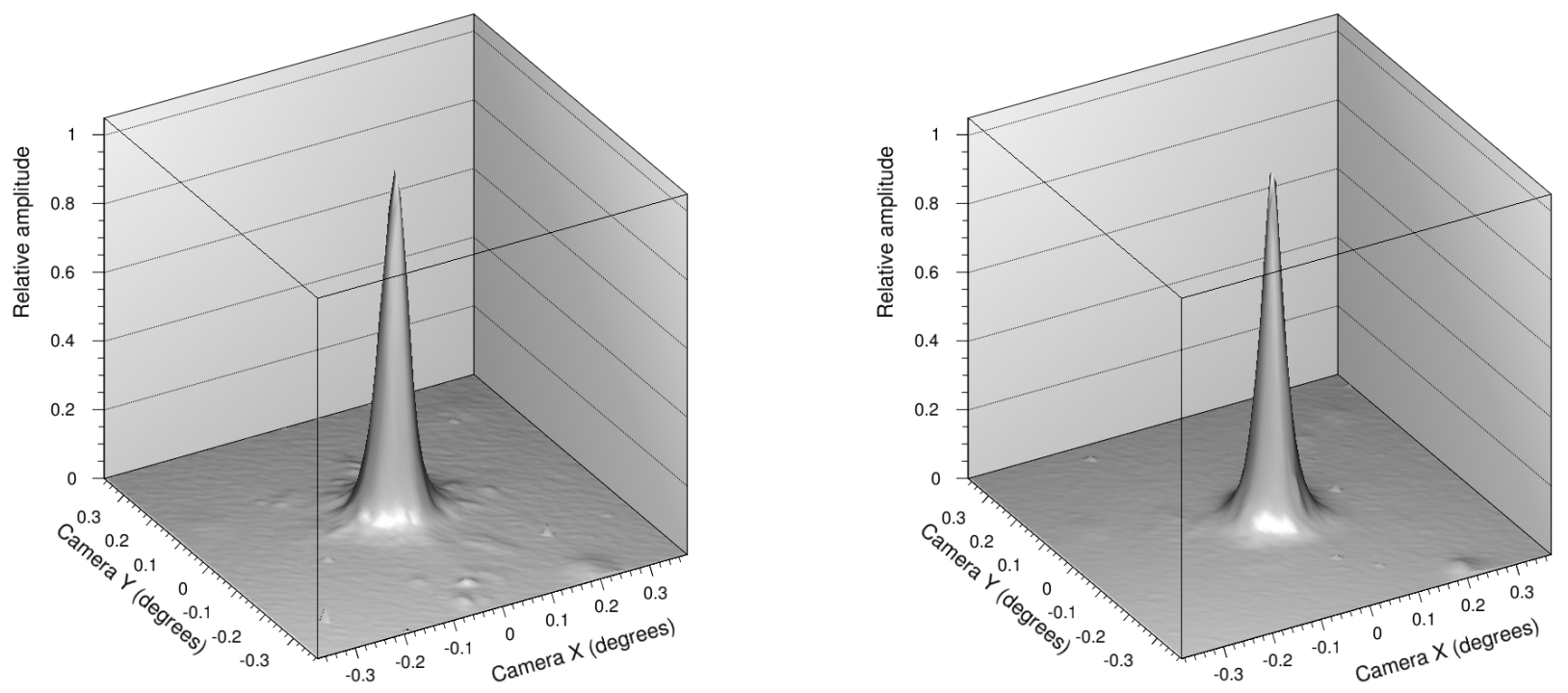

Figure 13: Optical point spread function for the two MAGIC telescopes (MAGIC-I left, MAGIC-II right). The image of the star called Menkalinan taken with the SBIG ${ }^{\circledR}$ camera at a zenith distance of 16 degrees.

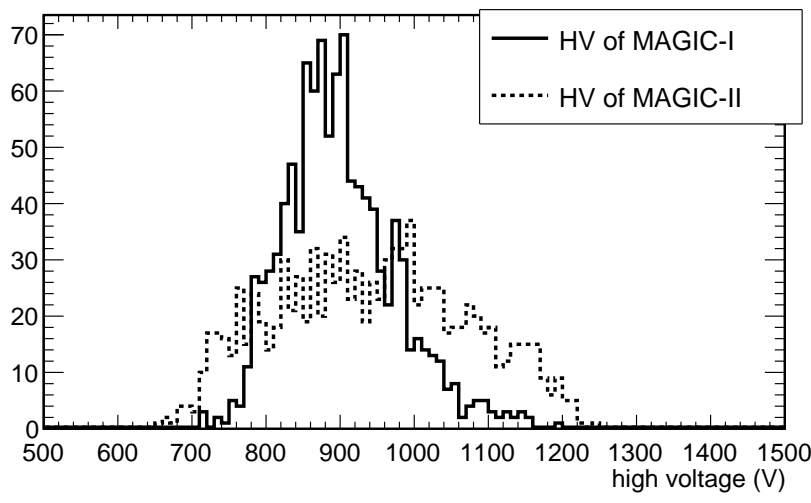

Figure 14: Distribution of the high voltages (HVs) applied to PMTs in MAGIC-I and MAGIC-II cameras after the charge flatfielding procedure. See text for details. The highest voltage that can be applied to the MAGIC PMTs is $1250 \mathrm{~V}$.

cess. We measured such spread in the PMTs for MAGIC I and found that it is about $30-50 \%$ (RMS), depending on the production line. The signal propagation chain introduces further differences in the gain: the optical links as well as the PIN diodes of the receivers mainly contribute to them. For the purpose of easier calibration of the signals and consistent saturation effects, the HVs applied to PMTs are adjusted such that the resulting signal from calibration pulses (equal photon number at the entrance of the PMTs) is equal in readout counts in all pixels when extracted after the digitization process. The resulting HV distribution for MAGIC-I and MAGIC-II cameras can be seen in Fig. 14. The distribution of the MAGIC-I camera is narrower. This is due to the fact that during the construction of the MAGIC-I camera the PMTs were divided into two categories, high and low gain ones (see Section 3.1). The high-gain PMTs are attenuated in the PMT base, which reduces the spread of the resulting gain distribution and consequently the spread of the HV distribution. The quality of the HV flatfielding can be seen in Fig. 15.

\subsection{Trigger adjustments and validation}

One of the most relevant systematic uncertainties of the detector originates from the camera's inhomogeneous response to gamma rays. The inhomogeneity of the recorded Cherenkov pulses can come from different gains in the electronic chain, different electronic noise levels or different levels of the night sky background light (presence of stars in the field of view). While the recorded pulses can be calibrated and flatfielded on the analysis level, the trigger inhomogeneity cannot be easily recovered. Therefore, a special attention is given to make sure all channels in the trigger are working well, the DTs are flatfielded and all multiplicity combinations in the L1 trigger are properly functioning. During the commissioning there were two major tasks concerning the L1 trigger: A) validation of all next neighbor multiplicities and B) the L0 delays are adjusted to assure that the time distribution of the Cherenkov photons in the focal plane of the telescope is 


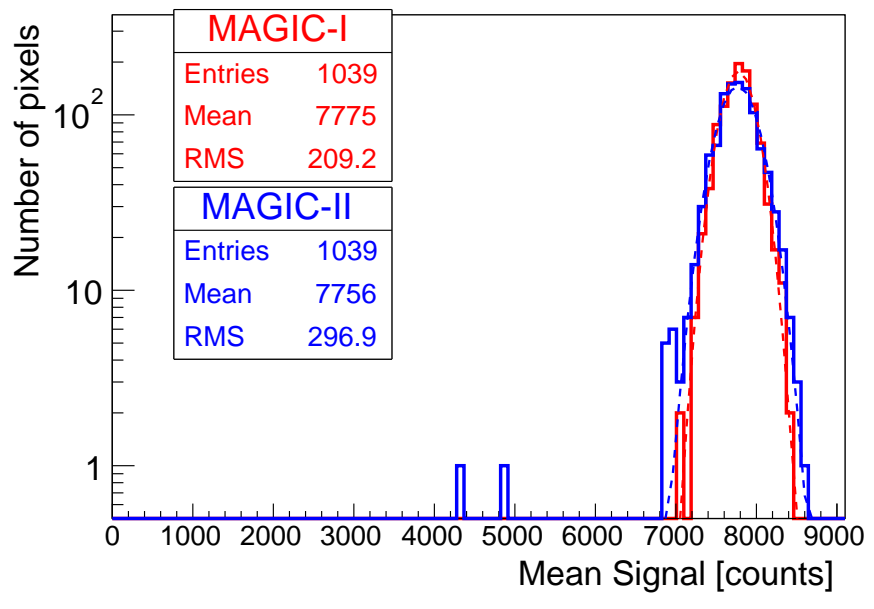

Figure 15: Charge distribution (mean and RMS) of the calibration pulses in the MAGIC-I (red) and MAGIC-II (blue) cameras after HV flatfielding. Data from 10 October, 2012. Two pixels in MAGIC-II could not be flatfielded well because the gain is too low and could not be increased further. These two pixels still can be used in the analysis.

conserved at the L1 trigger level. Dedicated hardware and software have been built to test all multiplicities in short time. The L1 trigger systems of both telescopes have been extensively tested, hardware mistakes identified and repaired.

\subsubsection{Evaluation of the L1 trigger}

The L1 trigger was evaluated with HYDRA, a multithread C-program to test, adjust and monitor the L1 trigger. The program is running as a part of the MAGIC Integrated Readout (MIR) software, which is the slow control program to steer and monitor individual readout, trigger and calibration system components of the MAGIC telescopes (Tescaro et al. 2009). There are many trigger pixel combinations to test: 1653 for $2 \mathrm{NN}, 988$ for $3 \mathrm{NN}, 1311$ for $4 \mathrm{NN}$ and 2280 for $5 \mathrm{NN}$. To test the L1 macrocell multiplicities, signals in all trigger channels are injected. This can be done during the day thanks to the pulse injection system of the camera (see Sec. 3.1). The DTs are set below the injected signal for a particular pixel combination in every macrocell, the others are set high enough to ensure that they will not trigger. The rate of the macrocell is monitored to identify not working combinations. The algorithm checks all possible combinations sequentially but it runs in parallel for all 19 macrocells. To go from one combination to the next one, the DTs must be changed, which takes about $10 \mathrm{~ms}$ per pixel. The trigger rate of the macrocells is read every $10 \mathrm{~ms}$, which makes the scan fast. The procedure to test all possible trigger combination takes about $15 \mathrm{~min}$ allowing for regular monitoring of the trigger performance. During the commissioning of the upgraded system, about 20 channels in each telescope were found not working in the L1 trigger (mostly due to a bad soldering and faulty components), which then were repaired.

\subsubsection{LO delays and LO width adjustment}

The arrival times of the signals at the L1 logic as well as the widths of the L0 signals had to be adjusted. There is a trade-off between the L1 trigger gate (that depends on the widths of the L0 signals) and the accuracy of the arrival times adjustment. With no delay adjustment the time spread would have an RMS of 3-4 ns with some outliers up to $10 \mathrm{~ns}$. The spread of arrival times is due to differences in transit times of the electrons in PMTs (mainly because of different $\mathrm{HV}$ applied) and to differences in signal travel time through optical fibers, as well as slightly different response time of electronic components. One also needs to allow for some $2 \mathrm{~ns}$ differences in arrival time between individual channels due to the physics of the shower ${ }^{11}$. Without delay adjustment of individual channels the L1 trigger gate would, therefore, be at least $15 \mathrm{~ns}$ to secure maximal efficiency of the L1 coincidence trigger to gamma rays. A larger gate corresponds to a higher chance to receive an accidental trigger, and the accidental trigger rate is a factor limiting the energy threshold. The goal was, therefore, to keep the gate as low as possible by adjusting the arrival times between the channels. In the following we describe the approach we used.

In HYDRA, several algorithms were implemented to adjust the arrival times automatically. Since the transit time in the PMTs has a relevant contribution, the procedure must be performed with the flatfielded HVs and open camera, using calibration pulses (since they arrive simultaneously at the camera plane, see Section 3.5. The following algorithm was chosen to be the standard one: The adjustment is done in $3 \mathrm{NN}$ logic and a fixed L0 pulse width. In every macrocell, the central pixel of the macrocell is considered to be the reference channel. A 2D scan in delay times is performed with the two neighboring pixels (in a valid $3 \mathrm{NN}$ combination) and the delays are chosen to maximize the resulting $\mathrm{L} 1$ gate. An example of such $2 \mathrm{D}$ scans for 4 different macrocells with a particular 3NN combination between pixels $\mathrm{pA}, \mathrm{pB}$ and $\mathrm{pC}$ is shown in Fig. 16. The delay of pixel pA is kept constant whereas a scan in delays of pixels $\mathrm{pB}$ and $\mathrm{pC}$ is performed. The axes of the plots indicate pixel delays in ns. The yellow area marks the delay combinations that result in a valid L1 trigger. The blue cross in the center of the area corresponds to the chosen delays as the result of the scan. The signal width of L0 pulses is $5.5 \mathrm{~ns}$. The resulting L1 gates are in order of $(7 \pm 1)$ ns (Fig. 16). The procedure continues successively over the $3 \mathrm{NN}$ combinations of the macrocell in a spiral by keeping already adjusted delays fixed. At the end, a crosscalibration procedure between the macrocells is applied using the border channels that belong to more than one

\footnotetext{
${ }^{11}$ The time gradient can be up to 2 ns between neighboring pixels for high-energy showers with a large impact distance from the telescope.
} 
macrocell. We also apply an overall offset to the resulting L0 delays to align them at around 5 ns to minimize the trigger latency. The overall precision of the adjustment is $\pm 1 \mathrm{~ns}$. The procedure has been tested with different L0 signal widths finding that $5.5 \mathrm{~ns}$ FWHM is the shortest L0 signal that give robust and reproducible results with a high trigger homogeneity. The HYDRA-based procedure to adjust L0 delays (needed every time HVs are changed) takes about $15 \mathrm{~min}$, which is a substantial improvement compared to the former manual procedure that required several observing nights to finish. The resulting L0 delays are shown in Fig. 17.
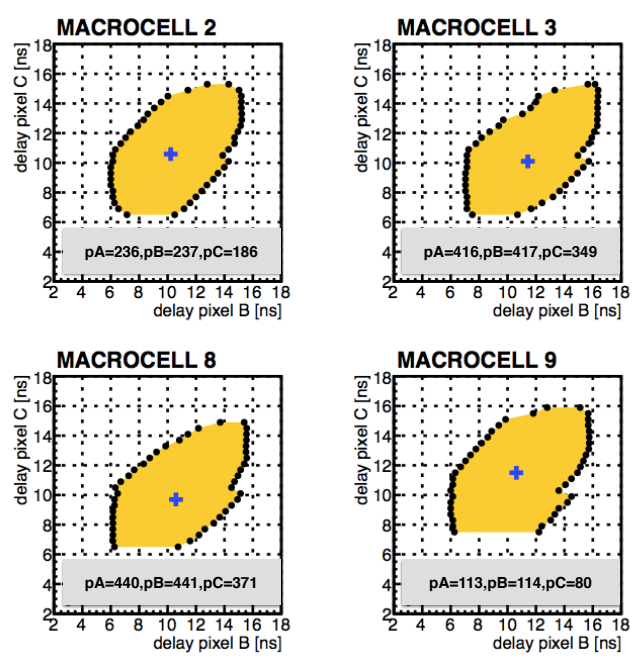

Figure 16: Example of the adjustement of the L0 delays between neighboring pixels of the $3 \mathrm{NN}$ logic. Shown are 4 macrocells with an example of $3 \mathrm{NN}$ combination between pixels $\mathrm{pA}, \mathrm{pB}$ and $\mathrm{pC}$. The result of the scan is shown by the blue cross. See text for details.

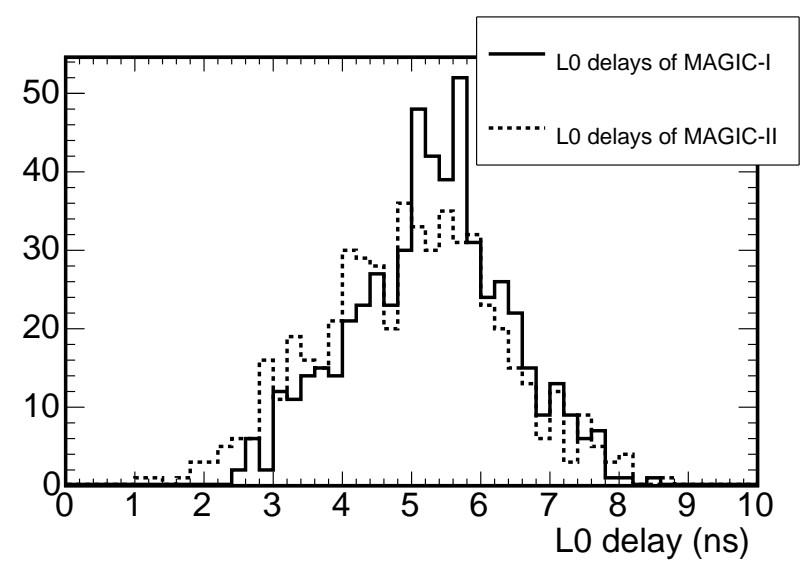

Figure 17: Distribution of the L0 trigger delays in MAGIC-I and MAGIC-II after applying the HYDRA optimization procedure.

\subsubsection{Discriminator threshold (DT) calibration}

The DTs of the trigger channels (L0 trigger) are adjusted such that the sensitivity of the channels is flat in terms of photon density of Cherenkov photons. This is achieved by means of a rate scan over the range of DTs for each trigger channel when firing calibration light pulses with a given photon density (e.g., equivalent to a mean of 100 photoelectrons (phe) per pixel in the PMTs of the camera). Then, for each trigger channel the required DT is determined such that half of the calibration pulses is accepted, and the other half is rejected. We then scale the DTs linearly to obtain a DT for a desired phe level. The standard DTs are set to be at a level of 4.25 phe. The distribution of the DTs for the two telescopes can be seen in Fig. 18. As there are some small differences between the analog (readout) and digital (trigger) signal chains, and the DT is applied to the amplitude, whereas the HV flatfielding is done for the integrated signal, there is some $\sim 15 \%$ RMS of the resulting DTs (pixel-to-pixel).

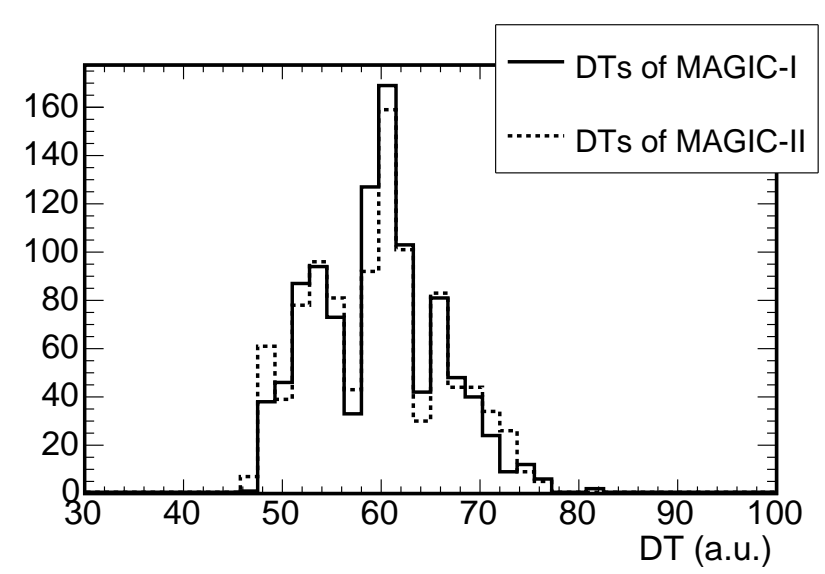

Figure 18: Distribution of the L0 discriminator thresholds (DTs) applied in MAGIC-I and MAGIC-II receiver boards after the charge flatfielding procedure. The L0 DTs correspond to a level of 4.25 phe.

\subsubsection{Individual pixel rate control (IPRC)}

During operation, the individual pixel rates (IPRs) are dominated by the night sky background. Bright stars inside the field of view (FoV) illuminate small areas of the camera and increase the IPR of the affected pixels. The flatfielded DTs may result in very different IPRs during the operation since a) the response of the PMTs is different for the calibration pulses for which the DTs are calibrated (fixed wavelength $355 \mathrm{~nm}$ ) and the night sky background (roughly a power law spectrum growing to red wavelengths) and b) the rates depend on the sky region the pixel is exposed to (e.g. it may contain stars, which would increase NSB fluctuations and, therefore, the IPR). During the commissioning, several algorithms and limits were 
tested and the following procedure established: As long as the IPRs are below 1.2 MHz (most of them being in the range of $300-600 \mathrm{kHz}$ ), no action is taken. For an IPR outside of this limit (due to a presence of stars), an IPR control software takes care of increasing the DT for the affected pixel in order not to spoil the resulting L1 telescope rate. Once the star is out of the FoV of the affected pixel, its IPR will be low because of the previously increased DT and once the IPR is below $100 \mathrm{kHz}$ the IPR control (IPRC) software resets the DT to the original value. This procedure ensures a flexibility for different NSB levels and takes care of the stars in the FoV while keeping the energy threshold low and most of the DTs flatfielded. It is important to keep the DTs flatfielded to ensure a good matching between the data and Monte Carlo simulations, where it is assumed that the DTs are identical for all the pixels.

\subsubsection{Adjusting the operating point of the trigger}

Rates scans have been performed at clear nights at low zenith angles to determine the trigger rate as a function of the DTs in phe. Mono (L1) trigger rate scans as well as stereoscopic (L3) trigger rates scans have been performed for several night and the performance has been shown to be stable. An example of the rate scans is shown in Fig. 19. One can see the steep slope of the rate at low DTs, where the rate is dominated by the noise coincidence. At higher DTs, the trigger rate is dominated by the rate of the cosmic ray showers. One can see that the coincidence trigger (L3 trigger) strongly suppresses the chance coincidence triggers. The operating point for MAGIC has been chosen to be around 4.5 phe, resulting in a stereo rate of around $280 \mathrm{~Hz}$, of which about $40 \mathrm{~Hz}$ are accidental triggers.

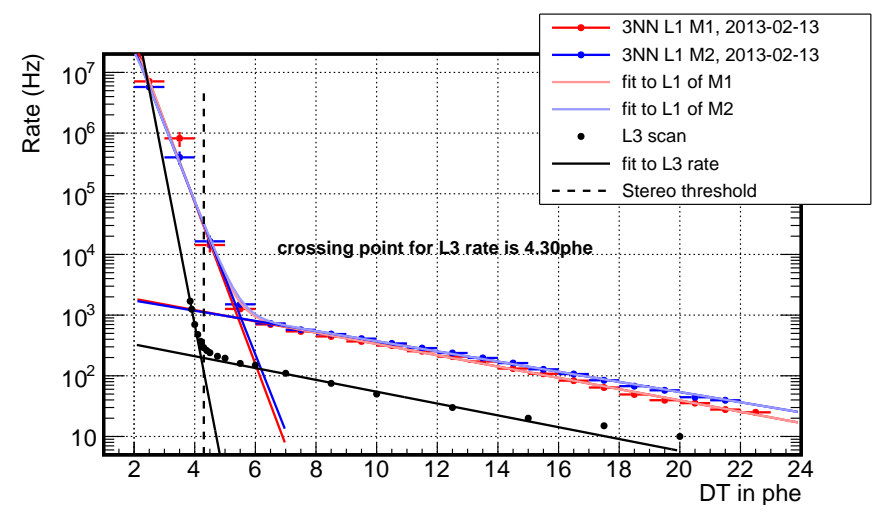

Figure 19: Rate scans taken changing discriminator thresholds (DTs) to optimize the operating point of the MAGIC telescopes. Red (blue) points are L1 3NN rate scans taken with MAGIC-I (MAGIC-II) telescopes and the lines are analytical fits to them. The black points correspond to the measured stereoscopic rate of the system, and the black lines are fitted functions. The operating point has been chosen to be around 4.5 phe per channel.
When pointing to new observation targets, it takes several seconds for the IPRC to adjust the DTs to the NSB light in the FoV. In the commissioning of the system we noted that, during these short periods, the resulting stereo trigger can be very high $(5-100 \mathrm{kHz})$, being dominated by accidental triggers. To avoid possible saturation of the data acquisition with very high trigger rates, which may lead to data corruptions and interruption of data taking procedure, a trigger limiter was installed inside the prescaler ${ }^{12}$ of each telescope. The trigger limiter evaluates the overall trigger rate of a telescope every $10 \mathrm{~ms}$ and blocks triggers to the readout for the following $10 \mathrm{~ms}$ in case the rate is above a programmable limit. The trigger limiter is configured to block the trigger rates above $1000 \mathrm{~Hz}$.

\subsection{DAQ performance}

The total acquisition rate that the DAQ has to guarantee is the sum of the cosmic trigger rate provided by the L3 trigger $(\sim 280 \mathrm{~Hz}$, see section 5.3.5) plus the contribution of the diagnostic calibration and pedestal triggers $(25+25 \mathrm{~Hz})$ that are issued interleaved to the physical triggers. During the commissioning of the system we adjusted various parameters of the DAQ program such as the number of cores used, loads between different threads, DAQ buffer length etc. The total sustainable acquisition of the DAQ system is currently $\sim 800 \mathrm{~Hz}$ (CPU limited, the writing speed being $\sim 1.1 \mathrm{kHz}$ ), more than double than the actual data taking rate.

The CPU overhead is particularly demanding because besides the event building, the data integrity check and the data storage, the DAQ performs two further actions: the DRS4 raw data correction and the extraction of the online data check values. The raw data correction (see Section 3.3.3 is a particularly demanding task because it involves the manipulation of every single digitized data sample from the readout, that has to be pedestal-subtracted using the specific single DRS4 capacitor average pedestal value (Sitarek et al. 2013).

As discussed Section 3.3.1, the receiver boards of the upgraded system allow a very precise control of the trigger rate even when the light conditions change (IPRC of the L0 discriminators). Nevertheless, besides providing this sustainable average acquisition rate, the DAQ has to guarantee a certain tolerance to sudden increase of the acquisition rate 13 This capability is guaranteed-without event loss - by the DAQ program design, which relies on a volatile memory ring-buffer bridging the events to the disk. The event buffer is 10,000 events deep, or $10 \mathrm{~s}$ in time at a trigger rate of $1 \mathrm{kHz}$.

\footnotetext{
12 The prescaler board is used in the MAGIC telescope to select and prescale the triggers coming from the individual trigger sources before issuing a trigger signal to the readout system.

${ }^{13}$ Temporary increases of the trigger rate are typically determined by uncontrolled factors like car-flashes from the astronomers of the observatory, but might be potentially also due to particularly high gamma-ray fluxes from still unknown physics phenomena.
} 
While running, the DAQ also feeds the online analysis program dubbed MOLA (see Tescaro et al. 2013, and Section 6.

\section{The online analysis client}

A real time data analysis is an important part of the success of the IACT experiment. Most of the extragalactic and several Galactic very-high-energy sources are variable, some of them on time scales down to hours and minutes. A real time analysis of the data taken can provide essential time critical internal triggers to extend observation of flaring sources and alert other multiwavelength partners. The upgraded system allowed to develop a novel program to fulfill the task of analyzing the data as they are being taken, and provide online information to the observers of the measured gamma-ray flux and its time evolution: MAGIC Online Analysis (MOLA).

MOLA is a multithreaded $\mathrm{C}++$ program that runs simultaneously with the data acquisition software and acts as a receiving client of the event informations computed at the very moment the events are acquired by each telescope. In fact, as mentioned in the previous section, the DAQ software of MAGIC-I and MAGIC-II computes independently the signal and arrival time of each pixel of the telescope cameras. In this way the calculation of the image parameters and the latest steps of the data analysis are outsourced to a separate program on an independent computer.

The multithread program structure consists of three threads: two reading threads and one analyzing thread. The two reading threads are appointed to receive the data stream via TCP/IP from the two DAQs asynchronously and perform the non-stereo analysis steps. The main analysis thread is instead appointed to match the events from the two streams and perform the stereoscopic reconstruction.

Two independent TCP/IP streams are activated once the program starts, and each time the observation of a source is finished the current results are stored and the analysis reset. Each event stream contains the pixel signals (integrated charge) and the signal arrival time. The tasks of each reading thread are:

- Receive, interpret and temporarily store the relevant information from the event stream.

- Calibrate (flat-field) the gain and identify the dead pixels using interleaved calibration events.

- Check pedestal events to identify and interpolate noisy pixels with low signals (e.g. due to stars).

- Perform image cleaning to select pixels with a significant Cherenkov signal.

- Calculate image parameters, using standard MAGIC analysis software data structures (see Zanin et al. 2013).
- Estimate shower direction from a set of relevant parameters (image shape, orientation and time gradient along the major axis) by using the random forest method (Albert et al. 2008).

Single telescope events have to be combined to form stereo events in order to exploit the full potential of the stereo imaging technique. The coincidence events are recognized in each independent stream by means of the unique L3 trigger number.

The tasks which have to be accomplished in order to obtain high level analysis results are performed by the stereo analysis thread, and can be summarized as follow:

- Identify matching stereo events by means of the unique L3 trigger number.

- Calculate the event direction through a weighted average of the estimates from the two individual images.

- Calculate shower core impact point and impact parameters.

- Apply the background suppression by means of the hadronness gamma/hadron likelihood parameter (Zanin et al. 2013).

- Apply cuts and compute the signal excess plot with respect to the candidate source position.

- Produce sky-maps with $\gamma$-ray candidate events.

- Produce light curves (time evolution) of the measured $\gamma$-ray flux during the current observations.

Results are produced for two energy ranges: Low Energy (LE) and High Energy (HE) depending of the size of the event image in phe: The HE sample includes all events with at least 125 phe in each of the two telescopes; the rest of the events with at least 40 phe in each telescope constitutes the LE sample. For Crab Nebula low zenith angles $\left(<30^{\circ}\right)$ observations the median energies of these two samples are $\sim 110$ and $\sim 350 \mathrm{GeV}$, respectively. In the HE range, the sensitivity of the MOLA analysis have been estimated to be $1.0 \%$ of the Crab Nebula flux in $50 \mathrm{~h}$ observation time.

MOLA provides to the telescope operators high-level information about the currently observed astrophysical source such as signal excess plots and sky-maps with $\gamma$-ray candidate events, together with diagnostic information related to the signal calibration and the image parameters calculation. MOLA is commissioned to perform without data loss up to a rate of $600 \mathrm{~Hz}$. For higher data rates (not expected with the current setup), some events will be lost for the online analysis but the program will continue running with a reduced performance. 


\section{Conclusions}

A major upgrade of the MAGIC telescopes took place in the years 2011-2012. The major items were the installation of the new camera, the new trigger in the MAGIC-I telescope, the upgrade of the readout system to DRS4 and programmable receiver boards in both telescopes. The commissioning of the upgraded system successfully finished in October 2012, and the telescopes restarted regular operation. The main goals of the upgrade were an improvement of the sensitivity at low energies, unification of the hardware used, and reduction of down time due to technical problems. These goals were been successfully achieved, as an example the down time due to technical problems was $<5 \%$ in the first two years after the upgrade was finished. This is more than a factor of two better than in the years before the upgrade and at the level required for the new generation Cherenkov telescopes of the CTA observatory (Acharya et al. 2013). The expectations concerning the sensitivity and the stability of MAGIC were surpassed, see details in the second part of the paper, Aleksić et al. (2014).

\section{Acknowledgements}

We would like to thank the Instituto de Astrofísica de Canarias for the excellent working conditions at the Observatorio del Roque de los Muchachos in La Palma. The support of the German BMBF and MPG, the Italian INFN, the Swiss National Fund SNF, and the ERDF funds under the Spanish MINECO is gratefully acknowledged. This work was also supported by the CPAN CSD2007-00042 and MultiDark CSD2009-00064 projects of the Spanish Consolider-Ingenio 2010 programme, by grant 127740 of the Academy of Finland, by the Croatian Science Foundation (HrZZ) Project 09/176 and the University of Rijeka Project 13.12.1.3.02, by the DFG Collaborative Research Centers SFB823/C4 and SFB876/C3, and by the Polish MNiSzW grant 745/N-HESS-MAGIC/2010/0.

\section{References}

Acharya, B. S., Actis, M., Aghajani, et al., Mar. 2013. Introducing the CTA concept. Astroparticle Physics 43, 3-18.

Aharonian, F., Akhperjanian, A. G., Bazer-Bachi, A. R., et al., Oct. 2006. Observations of the Crab nebula with HESS. Astronomy \& Astrophysics457, 899-915.

Albert, J., Aliu, E., Anderhub, H., et al., Apr. 2008. Implementation of the Random Forest method for the Imaging Atmospheric Cherenkov Telescope MAGIC. Nuclear Instruments and Methods in Physics Research A 588, 424-432.

Aleksić, J., Alvarez, E. A., Antonelli, L. A., Antoranz, P., Asensio, M., et al., 2014. The major upgrade of the MAGIC telescopes, Part II: The achieved physics performance using the Crab Nebula observations, submitted.

Bartko, H., Goebel, F., Mirzoyan, R., Pimpl, W., Teshima, M., Aug. 2005. Tests of a prototype multiplexed fiber-optic ultra-fast FADC data acquisition system for the MAGIC telescope. Nuclear Instruments and Methods in Physics Research A 548, 464-486.
Biland, A., Garczarczyk, M., Anderhub, H., et al., 2008. The Active Mirror Control of the MAGIC Telescopes. In: 30st ICRC, Merida. Vol. 3 of International Cosmic Ray Conference. pp. 1353-1356.

Bitossi, M., Paoletti, R., Tescaro, D., 2014. Ultra-fast sampling and Data Acquisition using the DRS4 Waveform Digitizer, in preparation.

Borla-Tridon, D., Goebel, F., Fink, D., et al., Jul. 2009. Performance of the Camera of the MAGIC II Telescope. In: 31st ICRC, Lodz. International Cosmic Ray Conference.

Carmona, E., Coarasa, J. A., Barcelo, M., for the MAGIC Collaboration, Jul. 2009. A Flexible High Demand Storage System for MAGIC-I and MAGIC-II using GFS. In: 31st ICRC, Lodz. International Cosmic Ray Conference.

Daum, A., Hermann, G., Heß, M., et al., Dec. 1997. First results on the performance of the HEGRA IACT array. Astroparticle Physics 8, 1-11.

Doro, M., 2012. Reaching the lowest energy threshold of groundbased cherenkov telescopes with magicstereo: A goal achieved. Nuclear Instruments and Methods in Physics Research Section A: Accelerators, Spectrometers, Detectors and Associated Equipment 692 (0), 201 - 207, 3rd Roma International Conference on Astroparticle Physics.

Doro, M., Bastieri, D., Biland, A., Dazzi, F., Font, L., Garczarczyk, M., Ghigo, M., Giro, E., Goebel, F., Kosyra, R., Lorenz, E., Mariotti, M., Mirzoyan, R., Peruzzo, L., Pareschi, G., Zapatero, J., Sep. 2008. The reflective surface of the MAGIC telescope. Nuclear Instruments and Methods in Physics Research A 595, 200-203.

Fomin, V. P., Stepanian, A. A., Lamb, R. C., Lewis, D. A., Punch, M., Weekes, T. C., May 1994. New methods of atmospheric Cherenkov imaging for gamma-ray astronomy. I. The false source method. Astroparticle Physics 2, 137-150.

Giavitto, G., 2013. Observing the VHE Gamma-Ray Sky with the MAGIC Telescopes: the Blazar B3 2247+381 and the Crab Pulsar.

Holder, J., Acciari, V. A., Aliu, E., et al., Dec. 2008. Status of the VERITAS Observatory. In: Aharonian, F. A., Hofmann, W., Rieger, F. (Eds.), American Institute of Physics Conference Series. Vol. 1085 of American Institute of Physics Conference Series. pp. 657-660.

Lorenz, E., Apr. 2004. Status of the $17 \mathrm{~m}$ MAGIC telescope. New A Rev.48, 339-344.

Mirzoyan, R., Lorenz, E., 1997. On the Calibration Accuracy of Light Sensors in Atmospheric Cherenkov Fluorescence and Neutrino Experiments. International Cosmic Ray Conference 7, 265.

Reichardt, I., Rico, J., Carmona, E., et al., Jul. 2009. The MAGIC Data Center. In: 31st ICRC, Lodz. International Cosmic Ray Conference.

Rodriguez Garcia, J., et al., 2013. CHECK CHECK CHECK. In: 33rd ICRC, Rio De Janeiro. International Cosmic Ray Conference.

Schweizer, T., Lorenz, E., Martinez, M., Ostankov, A., Paneque, D., Oct. 2002. The optical calibration of the MAGIC telescope camera. IEEE Transactions on Nuclear Science 49, 2497-2503.

Sitarek, J., Gaug, M., Mazin, D., Paoletti, R., Tescaro, D., Sep. 2013. Analysis techniques and performance of the Domino Ring Sampler version 4 based readout for the MAGIC telescopes. Nuclear Instruments and Methods in Physics Research A 723, 109-120.

Tescaro, D., Aleksic, J., Barcelo, M., Bitossi, M., Cortina, J., Fras, M., Hadasch, D., Illa, J. M., Martinez, M., Mazin, D., Paoletti, R., Pegna, R., for the MAGIC Collaboration, Jul. 2009. The readout system of the MAGIC-II Cherenkov Telescope. In: 31st ICRC, Lodz. International Cosmic Ray Conference.

Tescaro, D., López-Oramas, A., Moralejo, A., Mazin, D., Daniela Hadasch for the MAGIC Collaboration, Oct. 2013. The MAGIC telescopes DAQ software and the on-the-fly online analysis client. In: 33rd ICRC, Rio De Janeiro. International Cosmic Ray Conference.

Zanin, R., et al., 2013. MARS, the MAGIC analysis and reconstruction software. In: International Cosmic Ray Conference. International Cosmic Ray Conference. 